\title{
Lesão exantemática manifestada após intoxicação alcóolica aguda: relato de caso
}

\author{
Exanthematic lesion manifested after acute alcohol intoxication: case report \\ Lesión exantemática manifestada tras intoxicación alcohólica aguda: reporte de caso
}

Recebido: 05/09/2021 | Revisado: 11/09/2021 | Aceito: 12/09/2021 | Publicado: 13/09/2021

\author{
Bárbara Queiroz de Figueiredo \\ ORCID: https://orcid.org/0000-0003-1630-4597 \\ Centro Universitário de Patos de Minas, Brasil \\ E-mail: barbarafigueiredo@unipam.edu.br \\ Ana Beatriz Lara Melo \\ ORCID: https://orcid.org/0000-0003-2465-778X \\ Universidade de Itaúna, Brasil \\ E-mail: anabeatrizlaramelo@gmail.com \\ Antônio Ricardo Neto \\ ORCID: https://orcid.org/0000-0001-6647-9374 \\ Centro Universitário de Patos de Minas, Brasil \\ E-mail: antonioneto12@unipam.edu.br \\ Bruna Damas de Carvalho \\ ORCID: https://orcid.org/0000-0001-7770-3028 \\ Centro Universitário de Goiatuba, Brasil \\ E-mail: brunadamascvlh@gmail.com \\ Iuri Pimenta Oliveira \\ ORCID: https://orcid.org/0000-0002-9004-1513 \\ Centro Universitário de Patos de Minas, Brasil \\ E-mail: iuripimenta@unipam.edu.br \\ Sarah Laís Penido Machado \\ ORCID: https://orcid.org/0000-0003-1153-8458 \\ Universidade de Itaúna, Brasil \\ E-mail: sarahlaispenido@gmail.com
}

\begin{abstract}
Resumo
Introdução: Dos muitos transtornos relacionados ao álcool presentes em indivíduos encaminhados para departamentos de atendimento de emergência, a intoxicação alcoólica aguda é a mais frequente, sendo uma condição clinicamente prejudicial que geralmente ocorre após a ingestão de uma grande quantidade de álcool. Objetivo: explanar um caso de paciente que, após intoxicação aguda por álcool, apresentou lesões exantemáticas, principalmente na face, característica de rosáceas. Metodologia: Este artigo trata-se de um estudo de caso clínico com perspectiva qualitativa e descritiva, que consiste em uma pesquisa em que, em geral, ocorre com coleta direta de dados, cujo o pesquisador é o instrumento indispensável. Descrição do caso: Trata-se de paciente do sexo masculino, 24 anos, que, após ingesta excessiva de bebida alcóolica, com sintomas de mal-estar, astenia, sonolência, diminuição da acuidade visual, taquicardia, náusea, cefaleia, dilatação pupilar, fala arrastada, hipoglicemia e perda de controle de movimentos corporais, em que a terapêutica consistiu em hidratação venosa, infusão de soro glicosado e reposição de tiaminas. Além dessa sintomatologia, o paciente apresentou, em consonância ao prurido, lesões exantemáticas maculopapulares morbiliformes em face, conforme exposto pela Imagem 1, características de rosáceas. Conclusão: A intoxicação alcoólica aguda é uma condição clinicamente prejudicial que geralmente ocorre após a ingestão de uma grande quantidade de álcool. Pode se manifestar clinicamente de várias maneiras e ter efeitos comportamentais, cardíacos, gastrointestinais, pulmonares, neurológicos e metabólicos, bem como dermatológicos, conforme exposto pelo presente estudo.
\end{abstract}

Palavras-chave: Exantema; Intoxicação alcóolica; Etanol; Emergência.

\begin{abstract}
Introduction: Of the many alcohol-related disorders present in individuals referred to emergency care departments, acute alcohol intoxication is the most frequent, being a clinically harmful condition that usually occurs after drinking a large amount of alcohol. Objective: to explain a case of a patient who, after acute alcohol intoxication, had exanthematic lesions, especially on the face, characteristic of rosacea. Methodology: This article is a clinical case study with a qualitative and descriptive perspective, which consists of a research that, in general, takes place with direct data collection, in which the researcher is the indispensable instrument. Case description: This is a 24year-old male patient who, after excessive alcohol intake, with symptoms of malaise, asthenia, drowsiness, decreased visual acuity, tachycardia, nausea, headache, pupillary dilation, slurred speech, hypoglycemia and loss of control of body movements, in which the therapy consisted of intravenous hydration, infusion of glucose serum and replacement of thiamines. In addition to these symptoms, the patient presented, in line with the pruritus,
\end{abstract}


morbilliform maculopapular exanthematic lesions on the face, as shown in Image 1, characteristic of rosacea. Conclusion: Acute alcohol intoxication is a clinically harmful condition that usually occurs after drinking a large amount of alcohol. It can manifest itself clinically in several ways and have behavioral, cardiac, gastrointestinal, pulmonary, neurological and metabolic effects, as well as dermatological, as exposed in the present study.

Keywords: Exanthema; Alcohol intoxication; Ethanol; Emergency.

\section{Resumen}

Introducción: de los muchos trastornos relacionados con el alcohol presentes en los individuos remitidos a los servicios de urgencias, la intoxicación aguda por alcohol es la más frecuente, siendo una condición clínicamente dañina que suele ocurrir después de beber una gran cantidad de alcohol. Objetivo: explicar el caso de un paciente que, tras una intoxicación aguda por alcohol, presentó lesiones exantemáticas, especialmente en el rostro, características de la rosácea. Metodología: Este artículo es un estudio de caso clínico con perspectiva cualitativa y descriptiva, que consiste en una investigación que, en general, se realiza con recolección directa de datos, en la que el investigador es el instrumento indispensable. Descripción del caso: Se trata de un paciente masculino de 24 años que, tras ingesta excesiva de alcohol, con síntomas de malestar, astenia, somnolencia, disminución de la agudeza visual, taquicardia, náuseas, cefalea, dilatación pupilar, dificultad para hablar, hipoglucemia y pérdida de control. de movimientos corporales, en los que la terapia consistió en hidratación intravenosa, infusión de glucosa sérica y reposición de tiaminas. Además de estos síntomas, la paciente presenta, en consonancia con el prurito, lesiones exantemáticas maculopapulares morbiliformes en la cara, como se muestra en la Imagen 1, características de la rosácea. Conclusión: La intoxicación aguda por alcohol es una condición clínicamente dañina que generalmente ocurre después de beber una gran cantidad de alcohol. Puede manifestarse clínicamente de varias formas y tener efectos conductuales, cardíacos, gastrointestinales, pulmonares, neurológicos y metabólicos, así como dermatológicos, como se expone en el presente estudio.

Palabras clave: Exantema; Intoxicación alcohólica; Etanol; Emergencia.

\section{Introdução}

O etanol $(\mathrm{CH} 3 \mathrm{CH} 2 \mathrm{OH})$ é um composto hidrossolúvel que atravessa rapidamente as membranas celulares, resultando em um equilíbrio pronto entre as concentrações intra e extracelulares (Vonghia et al. 2008). Sua absorção ocorre principalmente no trato intestinal proximal, a saber, no estômago (70\%) e no duodeno (25\%), enquanto apenas uma pequena porcentagem ocorre no restante do trato intestinal. A álcool desidrogenase gástrica (ADH) é responsável por 10\% do metabolismo do álcool (denominado "metabolismo de primeira passagem") e tem importantes diferenças relacionadas ao gênero. Os $90 \%$ restantes do etanol ingerido são metabolizados em acetaldeído ao longo de três vias enzimáticas hepáticas em diferentes porcentagens: (1) ADH hepático (90\%), (2) sistema oxidante de etanol microssomal (8-10\%), e (3) catalase (0-2\%) (Addolorato et al. 2002).

O álcool é a substância de abuso mais amplamente usada. Embora apenas 7\% da população em geral beba muito, quase metade da população consome álcool regularmente. O uso excessivo de álcool é especialmente prejudicial para a faixa etária mais jovem e resulta em 30\% de mortes por ano em homens com idades entre 15 e 29 anos (Lopez-Caneda et al. 2018). O consumo excessivo de álcool é um modo popular de ingestão de álcool em adultos jovens. O Instituto Nacional de Alcoolismo e Abuso de Álcool (NIAAA) define o consumo excessivo de álcool como um padrão de consumo em que a concentração de álcool no sangue (CAS) da pessoa atinge 0,08\% ou mais. Este nível de alcoolemia é tipicamente alcançado quando os homens consomem 5 bebidas ou as mulheres consomem 4 bebidas em 2 horas (Yost, 2002).

Dos muitos transtornos relacionados ao álcool presentes em indivíduos encaminhados para departamentos de atendimento de emergência, a intoxicação alcoólica aguda é a mais frequente, sendo uma condição clinicamente prejudicial que geralmente ocorre após a ingestão de uma grande quantidade de álcool. Os achados clínicos geralmente presentes em indivíduos intoxicados por álcool são devidos ao efeito da ingestão aguda de álcool em diferentes órgãos e aparelhos. A intoxicação alcoólica aguda é capaz de causar várias alterações metabólicas, incluindo hipoglicemia, acidose láctica, hipocalemia, hipomagnesemia, hipoalbuminemia, hipocalcemia e hipofosfatemia (Lovinger et al. 2013). Aliado a isso, os efeitos cardiovasculares relacionados à intoxicação alcoólica aguda incluem taquicardia, vasodilatação periférica e depleção de 
volume; essas características podem contribuir para a indução de hipotermia e hipotensão. Outro possível efeito cardiovascular é "síndrome do coração de férias", caracterizado por taquiarritmias atriais ou ventriculares e novo início de fibrilação atrial após ingestão aguda de álcool (Sokolovsky et al. 2020).

O manejo de um paciente intoxicado ocorre principalmente no pronto-socorro e visa estabilizar o quadro clínico do paciente, dependendo de sua apresentação clínica (Quadro 1). A avaliação das vias aéreas e a observação do desenvolvimento da função respiratória devem ser feitas (Irwin et al. 2017). A prevenção da aspiração também é obrigatória; portanto, a colocação do paciente em uma posição lateral pode ser útil. Deve ser obtido acesso intravenoso e administrada solução de fluido intravenoso para hidratar o paciente e corrigir desequilíbrios eletrolíticos e hipoglicemia. Na prática clínica atual, uma solução intravenosa de protocolo contendo dextrose, magnésio, folato, tiamina e multivitaminas é usada (por exemplo, uma solução intravenosa pré-misturada de dextrose a 5\% e cloreto de sódio a 0,45\%, $2 \mathrm{~g}$ de sulfato de magnésio, $1 \mathrm{mg}$ de folato e $100 \mathrm{mg}$ de tiamina). Os medicamentos antieméticos podem ser úteis em pacientes com náuseas e / ou vômitos. Vômito prolongado pode levar a hiponatremia; isso não deve ser corrigido muito rapidamente, pois pode induzir uma mielinólise pontina central (Jacob et al. 2020).

Quadro 1: Gerenciamento de intoxicação aguda.

\begin{tabular}{|c|c|}
\hline $\begin{array}{l}\text { Estabilização do paciente e avaliação das vias } \\
\text { aéreas }\end{array}$ & $\begin{array}{l}\text { Observação da função respiratória; } \\
\text { Prevenção da aspiração; } \\
\text { Ventilação mecânica, se necessário; } \\
\text { Acesso intravenoso; } \\
\text { Correção da administração de solução intravenosa de hipoglicemia e } \\
\text { desequilíbrios eletrolíticos (dextrose + magnésio + folato + tiamina + } \\
\text { multivitaminas); } \\
\text { Drogas antieméticas. }\end{array}$ \\
\hline Sedação do paciente, se necessário & $\begin{array}{l}\text { Droperidol; } \\
\text { Haloperidol }\end{array}$ \\
\hline Aceleração da eliminação do etanol & $\begin{array}{l}\text { Restrições físicas (não recomendado); } \\
\text { Metadoxina (300-900 mg) }\end{array}$ \\
\hline
\end{tabular}

Fonte: Vonghia et al. (2008).

Em pacientes agitados e violentos, podem ser utilizadas substâncias sedativas, incluindo droperidol ou haloperidol, entretanto, deve-se ter em mente a possibilidade de uma interação farmacológica entre as drogas sedativas e o álcool que pode levar à depressão respiratória e hipotensão. Deve-se evitar o uso de contenção física para evitar que o paciente escape e / ou traumas físicos, dadas as preocupações éticas quanto ao seu uso; eles devem ser considerados apenas em condições extremas. Em alguns casos, ventilação mecânica e cuidados intensivos devem ser fornecidos (Vollrath et al. 2017). Uma droga específica que é útil no tratamento da intoxicação alcoólica aguda é a metadoxina, que é o par iônico entre o carboxilato de pirrolidona e a piridoxina (Martínez et al. 2002). O carboxilato de pirrolidona está envolvido no metabolismo de aminoácidos através da via da glutationa, facilita a síntese de ATP e evita a diminuição de ATP no cérebro e no fígado, segundo resultados de estudos com ratos intoxicados de forma aguda com etanol (Cederbaum, 2012).

Outrossim, a piridoxina aumenta a taxa de degradação metabólica do etanol, reduzindo assim os danos às funções celulares causados pelo acetaldeído, o primeiro metabólito no processo de eliminação do etanol. A metadoxina parece ser capaz de acelerar o metabolismo do etanol em ratos e humanos devido a vários mecanismos, incluindo um aumento na atividade da acetaldeído desidrogenase, depuração plasmática de etanol e acetaldeído e eliminação urinária de cetonas (Ruiz et al. 2001). Em animais, a metadoxina inibe o aumento de ésteres de ácidos graxos no fígado de ratos tratados com etanol, 
restaurando a relação entre as substâncias gordurosas saturadas e insaturadas. Além disso, a metadoxina é capaz de prevenir a depleção de glutationa, danos por peroxidação lipídica, deposição de colágeno e secreções de TNF alfa induzidas por álcool e acetaldeído em hepatócitos e células estreladas hepáticas (Reynaud et al. 2001). Sob essa perspectiva, o presente estudo tem como objetivo explanar um caso de paciente que, após intoxicação aguda por álcool, apresentou lesões exantemáticas, principalmente na face, característica de rosáceas.

\section{Metodologia}

Este artigo trata-se de um estudo de caso clínico com perspectiva qualitativa e descritiva, que consiste em uma pesquisa em que, em geral, ocorre com coleta direta de dados, cujo o pesquisador é o instrumento indispensável. O presente estudo contou com o aporte de trabalhos acadêmicos entre os anos de 2001 e 2021. Por meio do acesso às principais bases de dados, a saber: dados National Library of Medicine (PubMed MEDLINE), Scientific Electronic Library Online (Scielo), Cochrane Database of Systematic Reviews (CDSR), Google Scholar, Biblioteca Virtual em Saúde (BVS) e EBSCO Information Services, no período compreendido entre março e julho de 2021. O Termo de Consentimento Livre e Assistido (TCLE) foi assinado pelo paciente para coleta de dados neste estudo.

\section{Relato de Caso}

Trata-se de paciente do sexo masculino, 24 anos, que, após ingesta excessiva de bebida alcóolica, com sintomas de mal-estar, astenia, sonolência, diminuição da acuidade visual, taquicardia, náusea, cefaleia, dilatação pupilar, fala arrastada, hipoglicemia e perda de controle de movimentos corporais, em que a terapêutica consistiu em hidratação venosa, infusão de soro glicosado e reposição de tiaminas. Além dessa sintomatologia, o paciente apresentou, em consonância ao prurido, lesões exantemáticas maculopapulares morbiliformes em face, conforme exposto pela Imagem 1, características de rosáceas.

Imagem 1: Lesão exantemática em face.

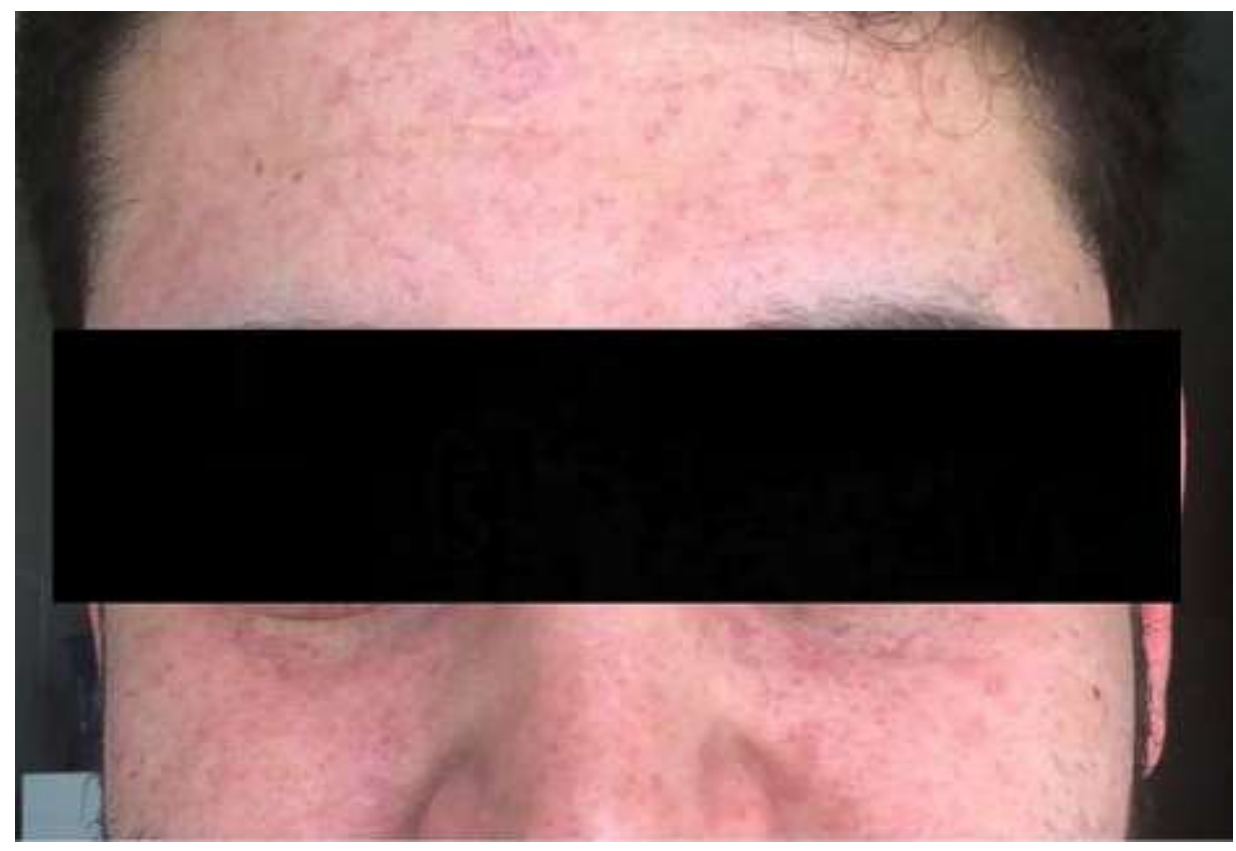

Fonte: Dados da pesquisa (2021).

\section{Discussão}


O álcool tem poder de dilatação de vasos sanguíneos, e, por conseguinte, de corroborar a vermelhidão e rubor cutâneo, bem como de prejudicar a permeabilidade intestinal, dificultando a capacidade de processamento de vitaminas C e E, causando inflamação e erupções cutâneas. Ademais, geralmente, bebidas açucaradas também têm potencial de causar inflamações na pele, e bebidas como o vinho, que são constituídas por salicilatos e histaminas, também podem corroborar reações alérgicas e sensibilidades cutâneas (Ceccanti et al. 2006).

Aliado a isso, o metanol, que é uma substancia relativamente barata e não taxativa por impostos sob as bebidas alcóolicas, tem sido utilizado criminosamente por sua adição em bebidas fabricadas clandestinamente, o que pode corroborar a uma alta toxicidade orgânica, podendo causar cegueira e até a morte. O máximo de metanol permitido nos destilados pela legislação brasileira é de $0,25 \mathrm{ml} / 100 \mathrm{ml}$ de álcool anidro, limite que geralmente é desrespeitado nas bebidas clandestinas. As bebidas são fabricadas com metanol misturado a $20 \%$ ao etanol. Como o cheiro é parecido, é transparente e também é inebriante, pode-se confundir com o etanol facilmente. O efeito tóxico do metanol resulta da sua metabolização em aldeído fórmico e deste em ácido fórmico. Para esses casos, o tratamento obriga à correção da acidose, causada por acumulação de ácido fórmico, com bicarbonato por via intravenosa, à diálise para eliminação do metanol intacto e do ácido fórmico e à inibição da formação dos metabólitos (Hanck et al. 2004).

\section{Conclusão}

A intoxicação alcoólica aguda é uma condição clinicamente prejudicial que geralmente ocorre após a ingestão de uma grande quantidade de álcool. Pode se manifestar clinicamente de várias maneiras e ter efeitos comportamentais, cardíacos, gastrointestinais, pulmonares, neurológicos e metabólicos, bem como dermatológicos, conforme exposto pelo presente estudo. O manejo da intoxicação alcoólica aguda tem como objetivo principal estabilizar o quadro clínico do paciente, acelerar a eliminação do álcool e definir e tratar todas as alterações clínicas citadas, e a metadoxina é uma droga eficaz e útil. Para pacientes com intoxicação alcoólica aguda, distúrbios relacionados ao álcool devem ser detectados e o paciente encaminhado a uma unidade de tratamento de álcool para um tratamento específico e personalizado.

\section{Referências}

Addolorato, G., Armuzzi, A. \& Gasbarrini, G. (2002). Alcoholism Treatment Study Group. Abordagens farmacológicas para o manejo da dependência do álcool. Eur Rev Med Pharmacol Sci., 6 (7), 89-97

Ceccanti, M., Attili, A. \& Balducci, G. (2006). Hepatite alcoólica aguda. J Clin Gastroenterol, 40 (8), 833-841

Cederbaum, A. I. (2012). Alcohol Metabolism. Clinics in Liver Disease, 16 (4), 667-685.

Hanck, C. \& Whitcomb, D. C. (2004). Pancreatite alcoólica. Gastroenterol Clin North Am., 33 (6), 751-765.

Irwin, C., Iudakhina, E., Desbrow, B. \& McCartney, D. (2017). Effects of acute alcohol consumption on measures of simulated driving: A systematic review and meta-analysis, Accident Analysis \& Prevention, 102 (17), 248-266.

Jacob, A. \& Wang, P. (2020). Intoxicação e cognição por álcool: implicações nos mecanismos e estratégias terapêuticas. Frontiers Neurosc., 14 (102), 1-9.

Lopez-Caneda, E., Cadaveira, F. \& Campanella, S. (2018). Editorial: alcoolismo no cérebro do adolescente e do jovem. Frente. Psychol., 9 (1), 2724-2730.

Lovinger, D. M. e Roberto, M. (2013). Efeitos sinápticos induzidos pelo álcool. Curr. Principal. Behav. Neurosci. 13 (3), $31-86$.

Martínez, M. C. L. D., Martínez, A. D. \& Salcedo, V. V. (2002). Eficácia da metadoxina no tratamento da intoxicação aguda por álcool. Journal of International Medical Research, 30 (1), 44-51.

Reynaud, M., Schwan, R., Loiseaux-Meunier, M. N., Albuisson, E. \& Deteix, P. (2001). Pacientes admitidos em serviços de emergência por embriaguez: usuários moderados de álcool ou bebedores nocivos? Am J Psychiatry, 158 (5), 96 - 99

Ruiz, G., Bucio, L. \& Correa, A. (2001). A metadoxina evita danos produzidos por etanol e acetaldeído em hepatócitos e células estreladas hepáticas em cultura. Pharmachol Res., 44 (8), 431-436. 
Research, Society and Development, v. 10, n. 12, e80101220257, 2021

(CC BY 4.0) | ISSN 2525-3409 | DOI: http://dx.doi.org/10.33448/rsd-v10i12.20257

Sokolovsky, A. W., Rachel, L., Micalizzi, L., White, H. R. \& Jackson, K. M. (2020). Alcohol and marijuana co-use: Consequences, subjective intoxication, and the operationalization of simultaneous use. Drug and Alcohol Dependence, 212 (1), 1-10.

Vollrath, M. \& Fischer, J. (2017). When does alcohol hurt? A driving simulator study, Accident Analysis \& Prevention, 109 (6), $89-98$.

Vonguia, L., Leggio, L., Ferrulli, A., Bertini, M., Gasbarrini, G. \& Addolorato, G. (2008). Intoxicação aguda por álcool. European Journal of Internal Medicine, 19 (1), 561-567.

Yost, D. A. (2002). Cuidado agudo para intoxicação por álcool: esteja preparado para considerar dilemas clínicos. Postgrad Med., 112 (21), 6-14. 